\title{
Evaluation of diaphragmatic fatigue in obstructive sleep apnoeas during non-REM sleep
}

Fabio Cibella, Giuseppina Cuttitta, Salvatore Romano, Vincenzo Bellia, Giovanni Bonsignore

\begin{abstract}
Background - Resistive load applied to the airways may induce diaphragmatic fatigue, and hypoxaemia has been shown to predispose to the development of fatigue. Inspiratory muscle fatigue may occur in patients with obstructive sleep apnoea syndrome (OSAS), as these patients repetitively develop both inspiratory loading and hypoxaemia. The results of previous studies on this topic are inconclusive, probably because of the methodological approaches used.
\end{abstract}

Methods - Six obese patients with OSAS underwent a polysomnographic study. The diaphragmatic pressure time index (PTI) was evaluated as an indicator of diaphragmatic contraction, and the mean frequency of the diaphragmatic electromyogram power spectrum (Fm) and the maximum relaxation rate of transdiaphragmatic pressure (MRR) as indices of a fatiguing diaphragm. A total of 119 randomly selected apnoeas (each including 5-13 occluded efforts) were analysed throughout the night in non-REM sleep to assess possible muscle fatigue due to the high pressure generation in each apnoea. A breath-by-breath withinapnoea analysis was performed on the first three pre-apnoeic breaths, on all the occluded efforts, and on the first three unoccluded breaths following the apnoea interruption. Possible fatigue development due to the cumulative effect of apnoeas over the night was also evaluated. Results - A progressive increase of $\mathrm{Fm}$ and MRR was found during the obstructive phase in all the subjects in the withinapnoea analysis. The overnight analysis did not show a reduction in either PTI, Fm, or MRR secondary to recurrent upper airway obstruction during the night.

Conclusions - No evidence of diaphragmatic fatigue or impaired diaphragmatic contraction was found either within each apnoea or throughout the whole night, despite the generation of high PTI values during the apnoeic occluded phases. It is concluded that diaphragmatic fatigue does not occur in OSAS during non-REM sleep.

(Thorax 1997;52:731-735)

Keywords: diaphragmatic fatigue, obstructive sleep apnoeas.
It has previously been demonstrated that an inspiratory resistive load applied to the airways may induce diaphragmatic fatigue, provided that some threshold relevant to the magnitude of the work performed by respiratory muscles and to the timing of the respiratory cycle is reached. ${ }^{1}$ Moreover, hypoxaemia has been shown to facilitate the development of fatigue of inspiratory muscles during inspiratory loading. ${ }^{2}$ Patients with obstructive sleep apnoea syndrome (OSAS) develop both inspiratory loading ${ }^{3}$ and hypoxaemia ${ }^{4}$ secondary to the recurrent upper airway obstruction. It has therefore been hypothesised that inspiratory muscle fatigue may occur during sleep in such patients.

The results published so far on this topic are controversial because of the different methodological approaches. Martin et $a \bar{P}$ were not able to find any evidence of fatigue when the diaphragmatic EMG power spectrum of patients with OSAS was evaluated during the night. Similarly, Vincken et $a l^{6}$ did not find electromyographic signs of diaphragmatic fatigue in OSAS even though the values of the diaphragmatic tension-time index exceeded the fatigue threshold at the end of apnoeas. Conversely, by evaluating maximal volitional occluded manoeuvres at the end of a night's sleep in patients with OSAS, Griggs et al found impaired inspiratory muscle contractility as demonstrated by a reduction in maximum relaxation rate of transdiaphragmatic pressure. ${ }^{7}$

On the basis of these conflicting studies, the fundamental question of the potential fatiguing role of obstructive apnoeas remains unanswered. Moreover, Griggs et al provided just an overall assessment of the phenomena, ignoring the longitudinal changes that may occur. Longitudinal changes may occur within each apnoea since chemical and mechanical stimuli progressively rise with the highest values at apnoea interruption. Other changes may occur throughout the night as an effect of the repetition of upper airway occlusive events.

The aim of this study was to evaluate the possible development of diaphragmatic fatigue in OSAS in non-REM sleep by comparing both the electromyographic and the mechanical approach to the breath-by-breath analysis of each apnoea and to the longitudinal analysis of subsequent apnoeas over the whole night.

Methods

Six obese patients (four men) of mean body mass index $37.2 \mathrm{~kg} / \mathrm{m}^{2}$ (range 30.4-48.4) aged 
Table 1 Pulmonary function and polysomnographic data for each subject

\begin{tabular}{lccclllrc}
\hline Subject & $\mathrm{Sex}$ & $\begin{array}{l}\mathrm{PaO}_{2} \\
(\mathrm{kPa})\end{array}$ & $\begin{array}{l}\mathrm{PaCO}_{2} \\
(\mathrm{kPa})\end{array}$ & $\begin{array}{l}\text { Baseline } \\
\mathrm{SaO}_{2}(\%)\end{array}$ & $\begin{array}{l}\text { No. of selected } \\
\text { apnoeas }\end{array}$ & $\begin{array}{l}\text { Mean (range) apnoea } \\
\text { duration (s) }\end{array}$ & $\begin{array}{l}\text { No. (range) of } \\
\text { occluded breaths }\end{array}$ & $\begin{array}{l}\text { Mean (range) } \\
\Delta \text { Sa } \mathrm{O}_{2}(\%)\end{array}$ \\
\hline CTR & $\mathrm{F}$ & 8.8 & 5.2 & 97 & 25 & $33.2(23-50)$ & $9(6-12)$ & $14.2(11-19)$ \\
DIM & $\mathrm{M}$ & 9.9 & 6.4 & 96 & 20 & $35.5(25-43)$ & $6.9(5-9)$ & $8.1(7-11)$ \\
$\mathrm{FLC}$ & $\mathrm{M}$ & 11.1 & 5.9 & 94 & 19 & $21.3(16-25)$ & $10.8(8-13)$ & $8.6(6-10)$ \\
FAS & $\mathrm{M}$ & 13.1 & 5.3 & 96 & 19 & $35.3(19-63)$ & $7.9(5-13)$ & $9.6(7-18)$ \\
GUG & $\mathrm{M}$ & 10.8 & 5.2 & 96 & 17 & $32.2(25-40)$ & $10.4(8-14)$ & $9.2(6-14)$ \\
PRO & $\mathrm{F}$ & 9.6 & 5.6 & 96 & 19 & $27.4(21-37)$ & $7.6(6-10)$ & $9.9(5-15)$ \\
Mean & & 10.5 & 5.6 & 95.8 & 19.8 & 30.8 & 8.8 & 9.9 \\
(SD) & $(1.5)$ & $(0.5)$ & $(1.0)$ & $(2.7)$ & $(5.5)$ & $(1.8)$ & $(2.2)$ \\
\hline
\end{tabular}

44.8 years (range $34-57$ ) with normal daytime respiratory function (mean forced expiratory volume in one second $\left(\mathrm{FEV}_{1}\right) 93.7 \%$ of predicted, range 81-109) and affected by OSAS (mean apnoea index 70.3 apnoeas/hour, range 38-103) were studied during nocturnal sleep after informed consent had been obtained.

The following signals were recorded on a chart recorder (Hewlett Packard 7758B, Hewlett Packard Co, Waltham, Massachusetts, USA) and on a magnetic tape recorder (Hewlett-Packard 3968A) for further analysis: electroencephalogram (C3A2 or C4A1 lead), electro-oculogram and chin electromyogram by surface electrodes for conventional sleep staging $;^{8}$ oronasal flow (V) by a light tightfitting face mask (approximately $80 \mathrm{ml}$ dead space, sealed to the face with a denture adhesive) with a pneumotachograph (Fleisch no. 1) connected to a differential pressure transducer (MP-45 Validyne, range $\pm 5 \mathrm{~cm} \mathrm{H}_{2} \mathrm{O}$, Validyne, Northridge, California, USA); pleural (Poes) and gastric (PGA) pressures by two balloon tipped catheters positioned in the lower third of the oesophagus and in the stomach, respectively, connected to two calibrated differential pressure transducers (MP45 Validyne, range $\pm 80 \mathrm{~cm} \mathrm{H}_{2} \mathrm{O}$ ), referenced to atmospheric pressure; electrocardiogram (ECG) by surface electrodes; diaphragmatic electromyogram (EMGdia) by an oesophageal electrode made of two silver rings $2 \mathrm{~mm}$ wide, spaced $18 \mathrm{~mm}$ apart, mounted at the distal end of a catheter anchored to the oesophagogastric junction by an inflated latex balloon: EMGdia was amplified and band pass filtered $(25-500 \mathrm{~Hz})$. The position of the PoEs catheter was adjusted following the occlusion technique proposed by Baydur et al..$^{9}$ A perforated tube, close to the outside edge of the face mask, was connected to a mass spectrometer (Centronic MGA 200, Centronic Ltd, UK) to detect possible air leaks from the mask. The reference sides of the PoEs and PGA manometers were adjusted in length to balance the opposite sides of the transducers and the balance was checked. ${ }^{10}$ Maximal inspiratory pleural and gastric pressures were obtained in all subjects in the supine position, before sleeping, during a maximal static inspiratory effort against a closed upper airway at functional residual capacity using a mouthpiece as suggested by Laporta and Grassino ${ }^{11}$ and maximum static inspiratory transdiaphragmatic pressure (maxPDI) was calculated. A total of 119 apnoeas, each including 5-13 occluded efforts, recorded in non-REM sleep during the study nights were analysed (17-25 apnoeas per patient equally spaced throughout the study night, table 1). The following signals were sampled at $1 \mathrm{~ms}$ intervals and stored in the mass memory of a computer (Digital Vax 8200, Digital Equipment Co, Maynard, Massachusetts, USA): EMGdia, PoEs, PGA, V', ECG. Transdiaphragmatic pressure (PDI) was calculated $(\mathrm{PDI}=\mathrm{PGA}-\mathrm{PoES})$. Inspiratory $(\mathrm{TI})$ and total breath cycle (Ttot) durations were measured on the PDI tracings ${ }^{6}$ for the three breaths immediately preceding the apnoea onset, all the occluded efforts, and the first three unoccluded breaths following the apnoea. For each breath the following parameters were calculated: peak inspiratory PDI (pPDI); mean inspiratory PDI ( $\overline{P D I})$; maximum PDI relaxation rate, evaluated as the maximum rate of the PDI decay after the inspiratory peak normalised by $\mathrm{pPDI}(\mathrm{MRR})$; diaphragmatic pressure time index (PTI) according to the formula:

$$
\mathrm{PTI}=\frac{\overline{\mathrm{P}} \mathrm{DI}}{\operatorname{maxPDI}} \times \mathrm{TI} / \text { Ttot } \%,
$$

where $\bar{P} D I$ is the mean PDI. For each inspiratory phase the sampled EMGdia signals were divided into continuous segments of $128 \mathrm{~ms}$. A Fast Fourier Transform routine was used to compute, for each segment, the mean frequency of the EMGdia power spectrum (Fm). For each segment the quality of the signal was checked by the computer software algorithms proposed by Sinderby et al. ${ }^{12}$ The EMGdia segments contaminated by the electrocardiographic QRS complexes were not analysed. The value of $\mathrm{Fm}$ for each breath was calculated as the mean of Fm values relevant to all the segments composing the breath.

The analysis of PTI, MRR and Fm data was performed both within each apnoea and throughout the night. The within-apnoea analysis was based on the breath-by-breath evaluation of all the pre-apnoeic, occluded, and postapnoeic breaths. The analysis of variance was used to evaluate the intra-apnoeic trend. The overnight analysis was performed for each subject by computing the time course of PTI, MRR, and Fm during the night and the slope of the linear regressions between the values of PTI, MRR and Fm measured in the occluded effort showing the highest inspiratory pPDI of each selected apnoea and its time during the night were calculated. The data management and all the statistical procedures were performed by the Systat statistical software package (SYSTAT Inc, Evanston, Illinois, USA). 


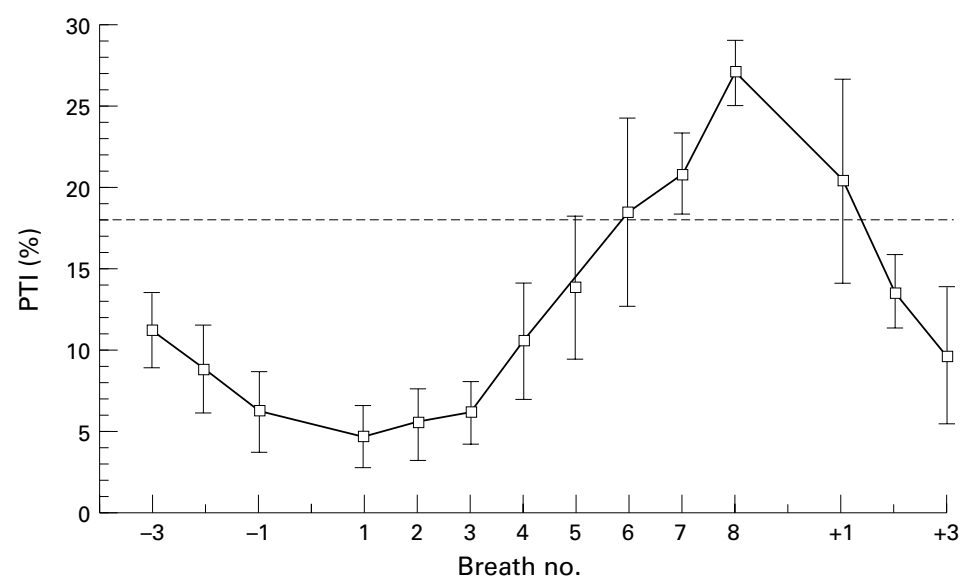

Figure 1 Time course of diaphragmatic pressure time index (PTI) during the three preapnoeic breaths, all the occluded efforts, and during the first three post-apnoeic breaths, for a representative subject. Each point represents the mean (SD) of the values relevant to corresponding breaths (or occluded efforts) over all the examined apnoeas. On the $x$ axis breath numbers are shown: $-3,-2,-1=$ third to last, second to last, and last preapnoeic breaths; $1-8=$ occluded efforts; $+1,+2,+3=$ first, second, and third postapnoeic breaths. The horizontal line shows the $18 \%$ fatigue threshold for PTI.

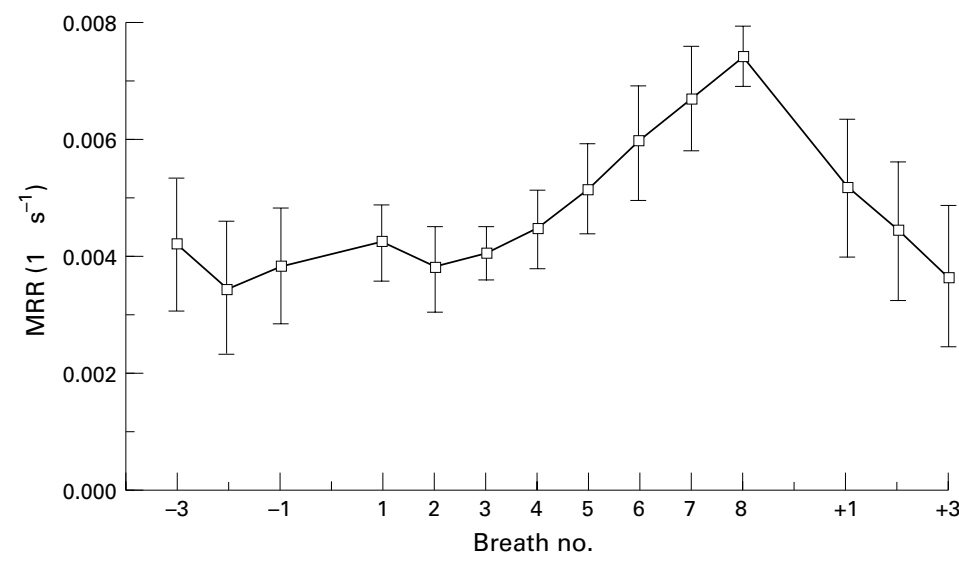

Figure 2 Time course of mean (SD) values of maximum transdiaphragmatic pressure relaxation rate normalised for transdiaphragmatic pressure inspiratory peak (MRR). The subject depicted in the figure is the same as in fig 1. For an explanation of the labels on the $x$ axis see legend to fig 1 .

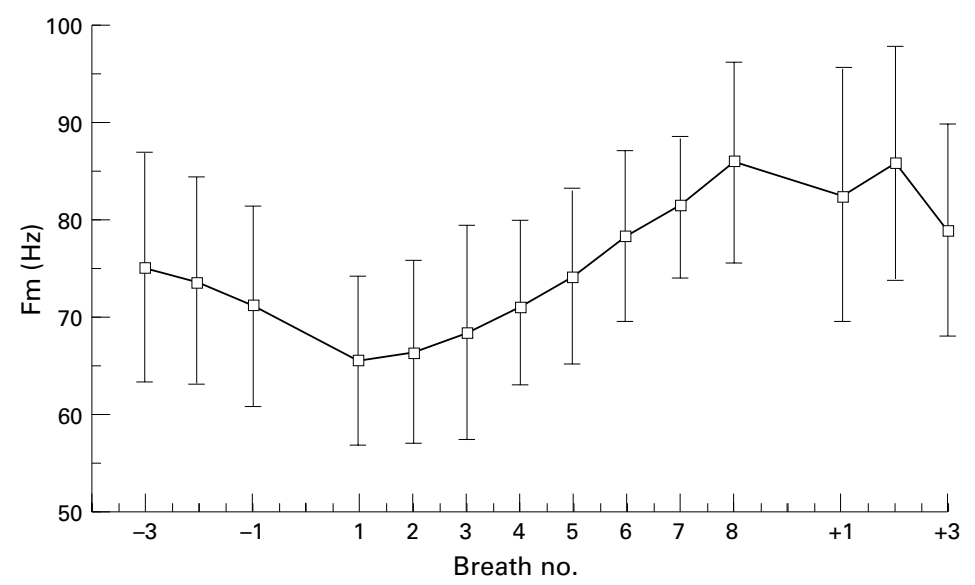

Figure 3 Time course of mean (SD) values of mean frequency of diaphragmatic electromyographic power spectrum (Fm). The subject depicted in the figure is the same as in figs 1 and 2. For an explanation of the labels on the $x$ axis see legend to fig 1 .
Results

The individual pulmonary function and polysomnographic data are listed in table 1. The mean maxPDI was $106.8 \mathrm{~cm} \mathrm{H}_{2} \mathrm{O}$ (range 90 148).

WITHIN-APNOEA ANALYSIS

All the subjects showed the same pattern of change in PTI, MRR, and Fm data. The results are presented as breath by breath analysis on the three pre-apnoeic breaths, all the occluded efforts, and on the first three post-apnoeic breaths for one representative subject in fig 1 (PTI), fig 2 (MRR), and fig 3 (Fm). PTI showed low values before the apnoea, progressively increasing during the occluded phase and reaching the highest values during the last occluded efforts (fig 1). It rapidly decreased in the post-apnoeic breaths, approaching the same values as the pre-apnoeic phase. During the occluded phase PTI reached or exceeded the value of 0.18 before apnoea termination. These peak PTI values were obtained at different time points - at the end of the apnoeic phase in some apnoeas and after several occluded breaths in others. MRR showed a slight increase during the apnoeic phase, reaching the highest value at the last occluded efforts (fig 2); this increase was significant in all but one subject (PRO). Fm showed a slight decline in the pre-apnoeic and early occluded phase and then increased towards the end of the apnoea; similar values were obtained in the post-apnoeic breaths (fig 3). In subject FAS Fm did not follow this pattern in the occlusive phase, showing no significant changes.

\section{OVERNIGHT ANALYSIS}

The trends in PTI, MRR, and Fm during the whole night are shown in fig 4 for all the subjects. For each variable each line represents the slope relevant to the occluded effort with the highest PDI value of all the evaluated apnoeas. The slopes of the three examined variables versus time of the night were positive or did not significantly decrease in all the subjects. No sign of reduction in the inspiratory efforts (decrease in PTI) or of changes in the indexes of diaphragmatic fatigue (decrease in MRR and/or Fm) were therefore found as an effect of the repeated occluded efforts during the night.

\section{Discussion}

Our results, based on both the mechanical and the electromyographic data, show that diaphragmatic fatigue does not occur as a consequence of OSAS in the range of severity of our subjects. We were not able to find any evidence of inspiratory muscle fatigue (in terms of reduction in Fm or MRR) either by breathby-breath analysis of individual apnoeas or as a cumulative effect of recurrent upper airway obstruction during the night.

Diaphragmatic fatigue is a temporary inability to generate a previously reachable PDI. The ratio between the high and the low com- 

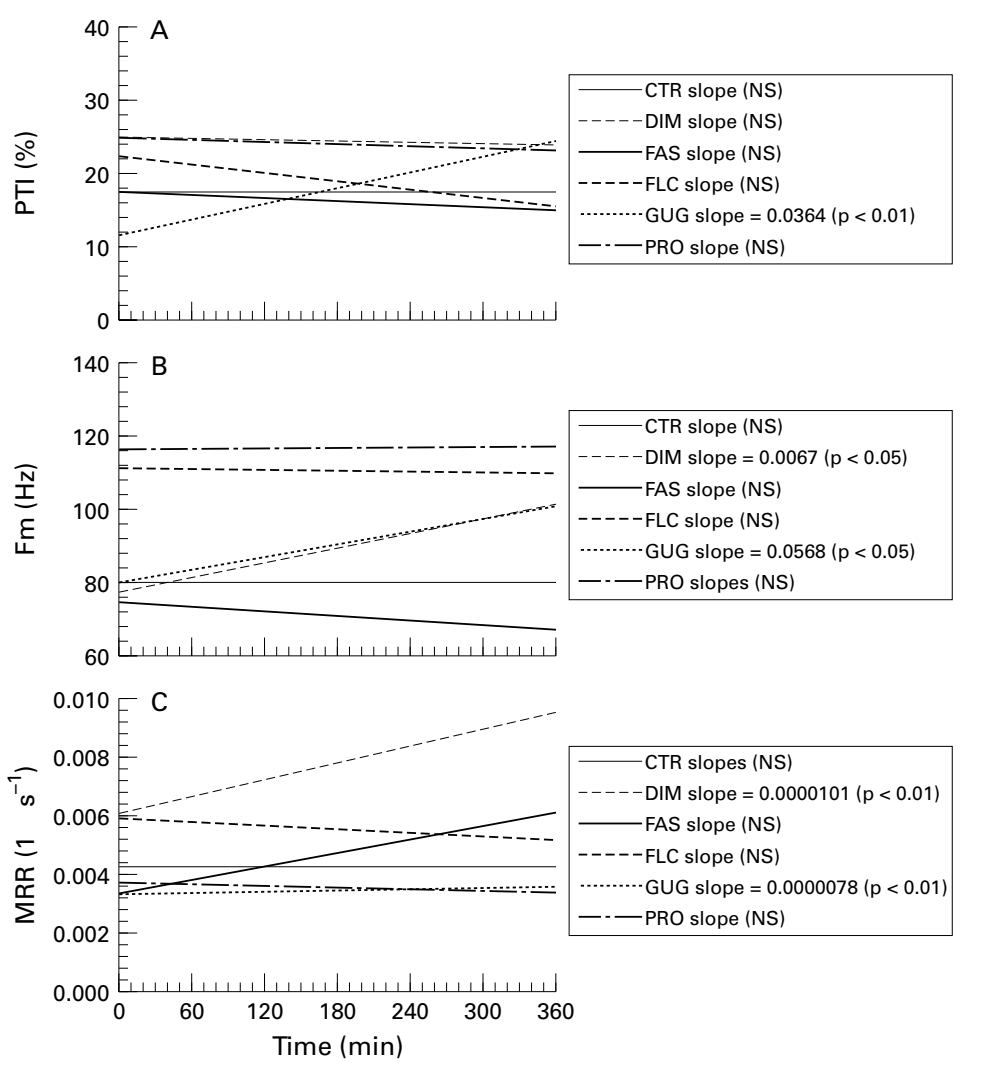

Figure 4 Slopes of the relationships between $(A)$ diaphragmatic pressure time index (PTI), (B) mean frequency of diaphragmatic electromyographic power spectrum (Fm), and $(C)$ maximum transdiaphragmatic pressure relaxation rate normalised for transdiaphragmatic pressure inspiratory peak (MRR) and time of the study night. The slopes were calculated on the values obtained from the occluded breath with the highest developed PDI of each analysed apnoea. On the $x$ axis the time of the study night is shown. All of the slope values were not significant or positive (see Results).

ponents of power spectrum $(\mathrm{H} / \mathrm{L})$ and Fm are indices able to demonstrate a shift in the EMG spectra from high to low frequencies. This kind of shift of the EMGdia power spectrum has been shown to be closely related to the early development of diaphragmatic fatigue. ${ }^{1314}$ Mechanical parameters have been used for the early detection of diaphragmatic fatigue and Esau $e t a l^{15}$ found that changes in the maximum relaxation rate of PDI may allow the prediction of diaphragmatic fatigue. This index was found to correlate well with changes in $\mathrm{H} / \mathrm{L}$. In fact, both indices showed a temporal relationship with muscle fatigue development, even though the maximum relaxation rate and $\mathrm{H} / \mathrm{L}$ appear to be determined by different biochemical mechanisms.

$\mathrm{H} / \mathrm{L}$ was used in previous investigations based on electromyographic signs of respiratory muscle fatigue. ${ }^{56}$ Conversely, we used Fm since it has been shown to be more stable, reproducible and sensitive to muscle fatigue than $\mathrm{H} / \mathrm{L} .{ }^{14}$ With regard to mechanical evaluation of fatigue in OSAS, a previous study was based on PDI maximum relaxation rate. We used the ratio between the latter index and pPDI to allow a breath-by-breath comparison to be made between mechanical and electromyographic indices during non-maximal non-volitional breathing manoeuvres. This normalisation is made necessary by the fact that the PDI re- laxation rate has been demonstrated to be linearly correlated to the peak PDI; ${ }^{16}$ since occlusive apnoeic efforts do not result in the development of maximum PDI values, the PDI relaxation rate increases as a result of a progressive increase in the developed PDI. This may obscure the possible tendency of PDI to decrease due to the developing muscle fatigue.

MRR is also influenced by the length of diaphragmatic muscle fibres and, as long as they shorten, MRR increases. ${ }^{17}$ However, during obstructive apnoeas fibres lengthen due to the reduction in lung volume. ${ }^{18}$ Moreover, the recruitment of different respiratory muscles may interfere with the measurement of MRR. The phase of PDI relaxation temporally coincides with the diaphragmatic post-inspiratory inspiratory activity (DIAPIIA). Its changes may influence MRR, but DIAPIIA abruptly decreases at the beginning of the occlusive apnoeic phase and its changes during this phase are minimal. ${ }^{19}$ It is therefore unlikely that DIAPIIA modifies MRR during the occlusive phase. Alternatively, the contraction of abdominal muscles during expiration may influence the MRR, distorting the PDI wave. Contraction of abdominal muscles occurs during the late expiratory phase; after that DIAPIIA is terminated, ${ }^{20}$ which is after the period during which MRR is measured. On this basis, no interference between the expiratory abdominal muscle contraction and the decay of PDI would be expected during sleep.

The time course of analysed variables (PTI, MRR and Fm) during apnoeas was quite similar for all the subjects. Our results concerning the apnoea pattern do not support the development of fatigue. This finding is in agreement with previous reports that have failed to demonstrate any decrease in $\mathrm{H} / \mathrm{L}$ ratio relevant to the inspiratory efforts in the occluded phase. ${ }^{56}$ In our study, during obstructive apnoeas, potentially fatiguing PTI values are reached only in the last occluded efforts, and apnoea termination prevents the maintenance of such PTI levels for sufficient time to develop fatigue. ${ }^{6}$

Thus, mechanical loading associated with occlusive apnoeic efforts appears to be so shortlived as not to be sufficient to induce fatigue. However, the results of Griggs et al raise the hypothesis of a cumulation over time, the effect of which could only be noticed at the end of the night. ${ }^{7}$ To test this hypothesis with the highest possible sensitivity we based our analysis on the breaths associated with the heaviest load - that is, with the highest developed PDI - occurring in the late apnoeic phase, although not necessarily associated with the last occluded effort. No subject (including DIM and PRO whose mean generated PDI corresponded to $81 \%$ and $62 \%$, respectively of maximum static PDI) showed any sign of diaphragmatic fatigue during the course of the night. The lack of agreement with the results of Griggs and coworkers may be explained in the light of different approaches to the evaluation of muscle contractility. The data of Griggs and coworkers were obtained by maximal voluntary manoeuvres, whereas our results are derived from 
non-maximal non-volitional occluded efforts during spontaneous sleep. This difference may be important since it has been shown that sleep loss may also affect muscle contractility. ${ }^{21} \mathrm{We}$ therefore infer that manoeuvres performed during wakefulness, as in the study of Griggs, may be influenced by sleep loss to a great extent, whereas events occurring during sleep are likely to be much less affected.

In conclusion, although obstructive apnoeas represent a challenge for respiratory muscles, neither their intrinsic load nor the cumulative effect appear to cause diaphragmatic fatigue whatever approach to its detection is used.

1 Bellemare F, Grassino A. Evaluation of human diaphragm fatigue. $\mathcal{F}$ Appl Physiol 1982;53:1196-206.

2 Jardim J, Farkas C, Prefaut C, Thomas D, Macklem PT, Roussos C. The failing inspiratory muscles under normoxic and hypoxic conditions. Am Rev Respir Dis 1981;124: oxic and $274-9$.

3 Martin RJ, Pennock BE, Orr WC, Sanders MH, Rogers RM. Respiratory mechanics and timing in occlusive sleep
apnea. $尹$ Appl Physiol 1980;48:432-7. apnea. F Appl Physiol 1980;48:432-7.

Coccagna G, Mantovani M, Brignani F, Parchi C, Lugaresi E. Continuous recording of the pulmonary and systemic arterial pressure during sleep in syndromes of hypersomnia with periodic breathing. Bull Eur Physiopathol Respir 1972; 8:1159-72.

5 Martin RJ, Fernandez E, Hudgel DW, P Hill. The association of sleep apnea to the high/low ratio of the diaphragmatic EMG (abstract). Am Rev Respir Dis 1982; 125:107.

6 Vincken W, Guilleminault C, Silvestri L, Cosio M, Grassino A. Inspiratory muscle activity as a trigger causing the airways to open in obstructive sleep apnea. Am Rev Respir Dis 1987;135:372-7.
7 Griggs GA, Findley LJ, Suratt PM, Esau SA, Wilhoit SC, Rochester DF. Prolonged relaxation rate of inspiratory muscles in patients with sleep apnea. Am Rev Respir Dis 1989;140:706-10

8 Rechtschaffen A, Kales A. A manual of standardized terminology, techniques and scoring system for sleep stages of human subjects. University of California, Los Angeles: Brain Information Service/Brain Research Institute, 1968

9 Baydur A, Behrakis PK, Zin WA, Jaeger M, Milic-Emili J. A simple method for assessing the validity of the esophageal balloon technique. Am Rev Respir Dis 1982;126:788-91.

10 Hudgel DW. Variable site of airway narrowing among obstructive sleep apnea patients. F Appl Physiol 1986;61: 1403-9.

11 Laporta D, Grassino A. Assessment of trans-diaphragmatic pressure in humans. F Appl Physiol 1985;58:1469-76.

12 Sinderby C, Lindström L, Grassino AE. Automatic assessment of electromyogram quality. $\mathcal{F}$ Appl Physiol 1995; 79:1803-15.

13 Gross DA, Grassino A, Ross D, Macklem PT. The EMG pattern of diaphragmatic fatigue. $\mathcal{F}$ Appl Physiol 1979;46: $1-7$

14 Schweitzer TW, Fitzgerald JW, Bowden JA, Lynne-Davies P. Spectral analysis of human inspiratory diaphragmatic electromyograms. F Appl Physiol 1979;46:152-65.

15 Esau SA, Bellemare F, Grassino A, Permutt S, Roussos C, Pardy RL. Changes in relaxation rate with diaphragmatic fatigue in humans. F Appl Physiol 1983;54:1353-60.

16 Esau SA, Bye PTP, Pardy RL. Changes in rate of relaxation of sniffs with diaphragmatic fatigue in humans. $\mathcal{F} A p p$ Physiol 1983;55:731-5.

17 Levy RD, Esau SA, Bye PTP, Pardy RL. Relaxation rate of mouth pressure with sniffs at rest and with inspiratory muscle fatigue. Am Rev Respir Dis 1984;130:38-41.

18 Aronson RM, Alex CG, Onal E, Lopata M. Changes in end-expiratory lung volume during sleep in patients with occlusive apnea. F Appl Physiol 1987;63:1642-7.

19 Cibella F, Marrone O, Sanci S, Bellia V, Bonsignore G. Expiratory timing in obstructive sleep apnoeas. Eur Respir f 1990;3:293-8.

20 von Euler C. Brain stem mechanisms for generation and control of breathing pattern. In: Handbook of physiology. The respiratory system. Section 3, Volume II. Bethesda, Maryland: American Physiological Society, 1986.

21 Chen H, Tang Y. Sleep loss impairs inspiratory muscle endurance. Am Rev Respir Dis 1989;140:907-9. 